\title{
BMJ Open The Moment Study: protocol for a mixed method observational cohort study of the Alternative Nicotine Delivery Systems (ANDS) initiation process among adult cigarette smokers
}

Jennifer L Pearson, ${ }^{1,2}$ Sabrina L Smiley, ${ }^{1}$ Leslie F Rubin, ${ }^{1,3}$

Andrew Anesetti-Rothermel, ${ }^{1}$ Hoda Elmasry, ${ }^{1}$ Megan Davis, ${ }^{1,4}$ Teresa DeAtley, ${ }^{1,2}$ Emily Harvey, ${ }^{1,5}$ Thomas Kirchner, ${ }^{6}$ David B Abrams ${ }^{1,2,7}$

To cite: Pearson JL, Smiley SL, Rubin LF, et al. The Moment Study: protocol for a mixed method observational cohort study of the Alternative Nicotine Delivery Systems (ANDS) initiation process among adult cigarette smokers. BMJ Open 2016;6:e011717. doi:10.1136/bmjopen-2016011717

- Prepublication history for this paper is available online. To view these files please visit the journal online (http://dx.doi.org/10.1136/ bmjopen-2016-011717).

Received 2 March 2016 Accepted 9 March 2016

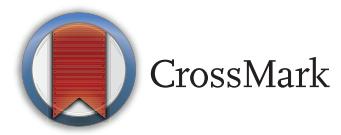

For numbered affiliations see end of article.

Correspondence to Dr Jennifer L Pearson; jpearson@truthinitiative.org

\section{ABSTRACT}

Introduction: Alternative Nicotine Delivery Systems (ANDS) such as e-cigarettes are battery-powered devices that aerosolize nicotine and other substances to simulate smoking without using tobacco. Little is known about the ANDS initiation process among adult smokers. The aims of this research are threefold to:

(1) examine how ANDS use affects cigarette use;

(2) examine how the immediate environmental and psychosocial contexts of cigarette and ANDS use vary within - and between - participants in general and by menthol preference and race; and, (3) examine participants' 'lived experience' of the subjective perceptions, meaning, influences and utility of cigarette and ANDS use.

Methods and analyses: This study's mixed method, 6-week longitudinal design will produce a detailed description of the ANDS initiation process among adult smokers $(N=100)$. Qualitative and quantitative data collection will include 3 weeks of: (1) ecological momentary assessment of patterns of cigarette/ANDS use, satisfaction, mood and craving; (2) geospatial assessment of participants' environment, including indoor and outdoor cigarette/ANDS norms and rules; (3) in-depth interviews about the meaning and utility of cigarette smoking and ANDS use; and, (4) saliva cotinine and exhaled carbon monoxide (CO) biomarkers. A diverse sample will be recruited with an equal number of menthol and non-menthol cigarette smokers. As the primary independent variable, we will investigate how ANDS use affects cigarette consumption. We will also examine how smokingrelated and ANDS-related rules and norms surrounding product use influence cigarette and ANDS product use, and how the subjective effects of ANDS use affect ANDS perceptions, beliefs and use.

Ethics and dissemination: This study was funded by the National Institute on Drug Abuse of the US National Institutes of Health (1R21DA036472), registered at ClinicalTrials.gov (NCT02261363), and approved by the Chesapeake IRB (Pro00008526). Findings will be disseminated to the scientific and lay

\section{Strengths and limitations of this study}

- Despite intense smoker interest in Alternative Nicotine Delivery Systems (ANDS) as a harm reduction or cessation aid, many smokers abandon ANDS after a short trial period and return to cigarette smoking.

- It is not possible to conduct randomised controlled trials testing the efficacy of ANDS use for smoking cessation in the current US funding and regulatory environment. Therefore, this mixed method intensive longitudinal design will use participants as their own controls to understand how ANDS use affects cigarette use, as well as how smoking and ANDS use rules and norms affect product choice.

- The study's innovative design combines ecological momentary assessment of mood, craving, satisfaction, cigarette and ANDS use; in-depth interviews; geotracking; and biospecimen collection to yield a detailed description of the first 2 weeks of an adult smoker's ANDS initiation experience.

- A limitation of this study design is that we only used first-generation 'cigalike' ANDS devices, which have been shown to be less reinforcing and deliver nicotine less effectively than secondgeneration devices.

community through presentations, reports and scientific publications.

Trial registration number: NCT02261363; Pre-results.

\section{INTRODUCTION}

Alternative Nicotine Delivery Systems (ANDS) are battery-powered devices that aerosolize nicotine and other substances to simulate 
smoking without using or igniting tobacco. An ANDS consists of a heating element and a repository that contains nicotine in a solution, usually with a variety of flavourings and other constituents. When the device is activated, the nicotine liquid is heated, producing an aerosol that is inhaled by the user. The term 'ANDS' encompasses a diverse category of products with different physical and performance characteristics, from disposable 'ciga-like' ANDS to large, customisable devices that more closely resemble consumer electronics than tobacco products.

There has been a rapid increase in lifetime and past 30-day adult use of ANDS in the USA. ${ }^{1}{ }^{2}$ The highest prevalence of ANDS use is among current and former cigarette smokers, ${ }^{3}{ }^{4}$ who most commonly report use to reduce cigarette consumption or to quit smoking. ${ }^{5-10}$ Available evidence suggest that a complete transition from combustible tobacco smoking to exclusive ANDS use will yield health benefits to the adult smokers who are otherwise unable or unwilling to quit using traditional smoking cessation methods. ${ }^{11-18}$ Despite intense smoker interest in ANDS as a harm reduction or cessation aid, it is notable that many smokers abandon ANDS after a short trial period and return to cigarette smoking, as suggested by the high prevalence of lifetime but not current ANDS use in this population. ${ }^{1} 319$

The 2009 Family Smoking Prevention and Tobacco Control Act gave the US Food and Drug Administration's Center for Tobacco Products (US FDA CTP) broad powers to regulate cigarettes, smokeless tobacco, and roll-your-own tobacco in the USA. ${ }^{20}{ }^{21}$ The US FDA CTP announced plans to expand its regulatory authority to ANDS in Summer 2014. However, little is known about the cognitions and behaviours leading to or sustaining ANDS use. ${ }^{22-25}$ Detailed, prospective information on the ANDS initiation period is necessary to understand the effect of ANDS on cigarette smoking among individual adult smokers and ultimately these devices' effect on public health. This exploratory study will contribute to the emerging field of ANDS research by identifying potential mechanisms predicting ANDS use, including how ANDS displace or are used concurrently with cigarettes. The aims of this research are threefold to: (1) examine how ANDS use affects cigarette use; (2) examine how the immediate environmental and psychological contexts of cigarette and ANDS use vary within- and between-participants by menthol preference and race; and, (3) examine participants' 'lived experience' of the subjective perceptions, meaning, influences, and utility of cigarette and ANDS use.

\section{METHODS AND ANALYSIS \\ Study design}

This 6-week intensive longitudinal study (the Mixed Method E-cigareTte Study, or 'Moment Study') will employ a mixed methods design to yield a detailed description of the ANDS initiation process among adult smokers who do not have plans to quit cigarette smoking in the next 30 days. Participants will complete 4 in-person visits, followed by an online follow-up survey 30 days after the final in-person visit (figure 1). The Moment Study's mixed method design features concurrent collection of multiple data streams, including: (1) ecological momentary assessment (EMA); (2) geotracking; (3) in-depth interviews; and, (4) biosamples. The EMA data will provide ecologically valid longitudinal data on cigarette smoking and ANDS use behaviour and associated mood, nicotine craving and satisfaction in real time. EMA data will be tagged with geo-location data that will be associated with the cigarette smoking and ANDS use rules and norms for each participant. Three weekly in-depth interviews will reveal participants' 'lived experience' of ANDS initiation, including expectancies, perceptions and cultural norms influencing cigarette and ANDS use. Exhaled carbon monoxide (CO) tests will be used to confirm recent cigarette smoking. Salivary cotinine will be used to assess nicotine consumption at the baseline and week 3 visits. A detailed schedule of assessments is presented in table 1.

In week 1 of the study, we will collect baseline information about participants' cigarette consumption, mobility patterns, and exposure to smoking rules and norms. At the week 2 visit, we will give participants a week's supply of ANDS (2 NJOY King 5-packs; 3.0\% nicotine) and ask them to try take at least three puffs daily over the course of the week. In week 3, we will replenish participants' ANDS supply (2 additional NJOY King 5-packs; 3.0\% nicotine) for ad lib use. Participants will be given regular or menthol disposable ANDS, depending on their regular brand cigarette flavour preference.
Figure 1 The Moment Study mixed method design. ANDS, Alternative Nicotine Delivery Systems.

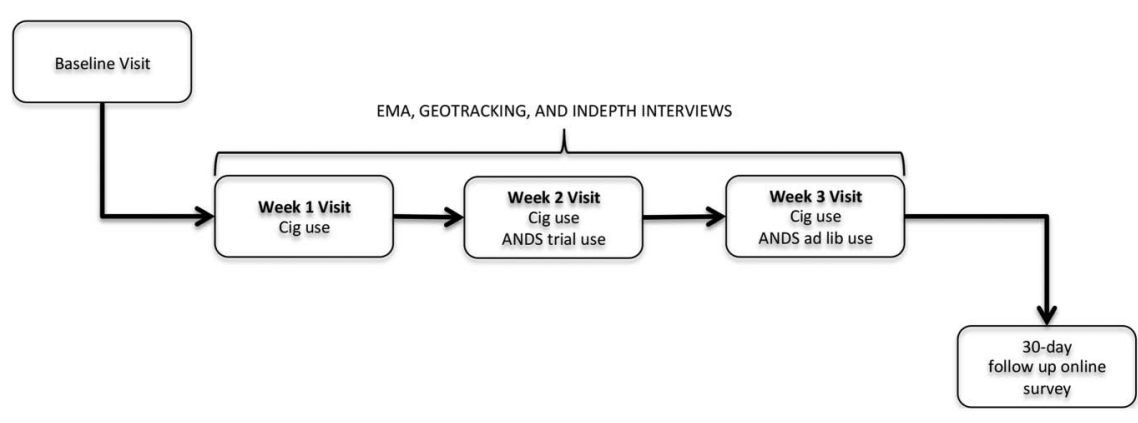


Table 1 The Moment Study schedule of assessments

\begin{tabular}{|c|c|c|c|c|c|c|}
\hline Variable & Mode & B & W1 & W2 & W3 & $\overline{F U}$ \\
\hline \multicolumn{7}{|l|}{ Primary outcomes } \\
\hline Cigarette use & Self-report EMA; Questionnaire; IDI & $\mathrm{X}$ & $\mathrm{X}$ & $\mathrm{x}$ & $\mathrm{X}$ & $\mathrm{X}$ \\
\hline ANDS use & Self-report EMA; Questionnaire; IDI & $\mathrm{X}$ & & $x$ & $\mathrm{X}$ & $\mathrm{X}$ \\
\hline \multicolumn{7}{|l|}{ Secondary outcomes } \\
\hline Motivation to quit cigarettes & Questionnaire & $\mathrm{X}$ & & & & $x$ \\
\hline Self-efficacy to quit cigarettes & Questionnaire & $\mathrm{X}$ & & & & $\mathrm{X}$ \\
\hline $24 \mathrm{~h}$ cigarette quit attempts & Questionnaire & $\mathrm{X}$ & & & & $\mathrm{X}$ \\
\hline \multicolumn{7}{|l|}{ Primary independent variables } \\
\hline Physical and social context of cigarette use & Questionnaire; IDI; Geotracking & $\mathrm{X}$ & $\mathrm{X}$ & $x$ & $\mathrm{X}$ & $\mathrm{X}$ \\
\hline Physical and social context of ANDS use & Questionnaire; IDI; Geotracking & $\mathrm{X}$ & $\mathrm{X}$ & $\mathrm{x}$ & $\mathrm{X}$ & $\mathrm{X}$ \\
\hline \multicolumn{7}{|l|}{ Covariates } \\
\hline Mood & Random EMA & & $\mathrm{X}$ & $x$ & $\mathrm{X}$ & \\
\hline Craving & Self-report and random EMA & & $\mathrm{X}$ & $\mathrm{x}$ & $\mathrm{X}$ & \\
\hline Satisfaction & Self-report EMA; Questionnaire & & $\mathrm{X}$ & $\mathrm{x}$ & $\mathrm{X}$ & $\mathrm{X}$ \\
\hline Forgot to report product use & Random EMA & & $\mathrm{X}$ & $\mathrm{X}$ & $\mathrm{X}$ & \\
\hline Sociodemographics & Questionnaire & $\mathrm{X}$ & & & & \\
\hline Tobacco/ANDS use behaviour & Questionnaire & $\mathrm{X}$ & & & & $\mathrm{X}$ \\
\hline Physical and mental health status & Questionnaire; IDI & $x$ & $x$ & & & \\
\hline Perceived stress & Questionnaire; IDI & $\mathrm{X}$ & $x$ & $\mathrm{x}$ & $\mathrm{X}$ & $x$ \\
\hline Social roles and self-concept & Questionnaire; IDI & $\mathrm{X}$ & $x$ & $x$ & $\mathrm{X}$ & $\mathrm{X}$ \\
\hline Alcohol use & Questionnaire & $\mathrm{X}$ & & & & \\
\hline Cigarette and ANDS social norms & Questionnaire; IDI & $X$ & $\mathrm{X}$ & $x$ & $\mathrm{X}$ & $\mathrm{X}$ \\
\hline Cigarette dependence & Questionnaire & $\mathrm{X}$ & & & & $\mathrm{X}$ \\
\hline ANDS dependence & Questionnaire & & & & & $\mathrm{X}$ \\
\hline ANDS purchasing & Questionnaire; IDI & $\mathrm{X}$ & & $x$ & $\mathrm{X}$ & $x$ \\
\hline Reason for ANDS use & Questionnaire; IDI & & $x$ & & & $\mathrm{X}$ \\
\hline Perceived harm/risk for addiction & Questionnaire; IDI & $\mathrm{X}$ & $x$ & $x$ & $\mathrm{X}$ & $x$ \\
\hline Cigarette and ANDS chemosensory experience & Questionnaire; IDI & $\mathrm{X}$ & $\mathrm{X}$ & $\mathrm{x}$ & $\mathrm{X}$ & $\mathrm{X}$ \\
\hline Cotinine & Biomarker & $\mathrm{X}$ & & & $\mathrm{X}$ & \\
\hline
\end{tabular}

ANDS, Alternative Nicotine Delivery Systems; EMA, ecological momentary assessment; FU, follow-up; GPS, global positioning system; IDI, In-depth interview.

Data will be collected over a 26-month period in 2014 and 2016. Due to the complexity of the study design, the study was piloted with three participants to test acceptability of study burden and functionality of the EMA text messaging and geotracking applications in Summer 2014. Pilot participants found the level of study burden acceptable and provided valuable feedback on the wording and specificity of EMA questions, which were integrated into the main study.

\section{Study population}

We will enrol up to 140 adults to achieve a final sample of 100 participants with complete data. We will attempt to recruit equivalent representation of menthol and non-menthol smokers and stratify by race/ethnicity such that our final sample will include 25 non-white menthol, 25 non-Hispanic white menthol, 25 non-white traditional smokers, and 25 non-Hispanic white traditional smokers to disaggregate the influence of menthol preference from tobacco use cultural norms or expectancies on ANDS use.

To be eligible to participate in the Moment Study, individuals must be aged 18 years or older, reside in the Washington DC, USA metro area, and be proficient in
English. Individuals must report daily smoking of at least eight cigarettes a day for the past 5 years, and must record an exhaled air carbon monoxide $(\mathrm{CO})$ level $>8$ parts-per-million (ppm) at the baseline visit. Individuals will be eligible if they do not intend to quit cigarette smoking in the next 30 days and are not currently taking a smoking cessation medication (eg, varenicline) or nicotine replacement therapy. To simplify EMA tobacco use reports (which focus only on cigarette use), individuals may not have smoked a little cigar/cigarillo, large cigar, or hookah more than five times in the past 30 days, and may not have used smokeless tobacco for the past 30 days. Additionally, individuals may not have used ANDS in the past 30 days, and must be interested in trying ANDS. To facilitate EMA data collection and geotracking, participants must have an iPhone or Android cellphone that allows installation of applications, use their cellphone daily, and have an unlimited text message plan. Individuals must be willing to travel to the data collection site (downtown Washington, DC) four times in 3 weeks and have no plans to spend the night out of town for more than five nights in the next 6 weeks. Finally, individuals may not: (1) be breastfeeding or planning to become pregnant; (2) have heart 
disease/uncontrolled blood pressure; (3) have psychosis/ suicidal thoughts; or, (3) be currently enrolled in an alcohol treatment program. All criteria will be assessed by self-report. Those who do not meet the criteria listed above will be ineligible for the study.

\section{Recruitment}

Participants will be recruited via public online postings (eg, Craigslist), paid advertisements (eg, Washington Metropolitan Area Transit Authority buses/stations, free weekly newspapers), and physical flyers. Recruitment documents will direct potential participants to an online screening survey optimised for viewing on computer, tablets and cellphones (http://www.ecigstudy.org) using the online BecomeAnEX Study System (BeXSS), a webbased clinical trials and survey management system created, owned and operated by Truth Initiative. The online screening survey will automatically identify eligible individuals and inform others that they are ineligible. A research assistant (RA) will call eligible individuals who have completed the screener to confirm eligibility and schedule a baseline office visit.

\section{Procedures}

All office visits will be conducted in a dedicated interview room in Truth Initiative's downtown Washington DC, USA office. During the baseline office visit, participants will complete the informed consent process and confirm current smoking status with an exhaled CO test. They will then complete the baseline survey and will schedule their remaining three in-person office visits, which will occur a week apart at approximately the same time of day. The RA will register participants' phones to receive EMA text messages and will train participants how to respond to the random EMA texts and selfinitiate tobacco use reports by initiating dummy random prompts and tobacco use reports. The RA will also install a free, temporary geotracking application on participants' cell phones to track their location over 3 weeks, and will teach participants how to turn off the geotracking application if they desire privacy. EMA and geotracking data will be automatically sent to a secure server, which an RA will monitor during the week to ensure data collection quality.

The weeks 1-3 office visit procedures are similar. Each visit will include: (1) a qualitative in-depth interview; (2) an RA-guided 'place' survey where participants geo-tag their personal mobility maps from the prior week with the smoking/ANDS rules and norms for locations they frequented; and, (3) compensation payment. During the week, an RA will text, call or email participants about their EMA and geotracking data to reduce missing data and increase data quality. During the weeks 1 and 2 visits, the RA will discuss the EMA data collection process with participants to identify barriers to data capture, discuss solutions and encourage continued performance. A salivary cotinine sample and $\mathrm{CO}$ reading will be collected at the baseline and week 3 visit. The geotracking application will be uninstalled at participants' final office visit at the end of week 3 .

Thirty days after their last contact with the study, an RA will send an email to participants with an embedded web link to the online follow-up survey. Like the screener, this survey has been optimised for viewing on a computer, tablet or phone. Participants will be emailed reminders three times within 7 days to complete the follow up survey. Participants who do not respond to the emails will receive phone calls from an RA encouraging them to complete the online survey. The RA may also administer the follow-up survey over the phone if the participant prefers. Though the Moment Study is not a treatment study, an RA will distribute a list of evidencebased free and low-cost cessation resources to any participant who indicates interest in cessation during the 3-week intensive monitoring period. As their final contact with the study, all participants will receive an email with encouragement to quit smoking and a list of evidence-based cessation resources regardless of their indicated interest in smoking cessation.

\section{Compensation}

We will provide participants with compensation for the time and effort they invest in the Moment Study. Cash payments will be provided at the baseline and weeks one, two, and three visits, and an emailed Amazon.com gift card will be provided as a final compensation for completion of the follow-up survey. Participants will also receive bonus payments for complying with the EMA study protocol. Participants who respond to at least 21 random texts per week will receive a bonus $\$ 5$ per week; those who respond to at least 30 random texts per week will receive a bonus of $\$ 10$ per week. Participants who self-initiate at least 41 cigarette/ANDS reports per week will receive a bonus of $\$ 5$; those who self-initiate at least 42 reports a week will receive a bonus of $\$ 10$. An RA will inform participants as to their progress towards earning the EMA completion bonuses during the week via phone call, text message, or email. Participants who complete all aspects of data collection, including the bonus for responding to at least 30 random EMA texts per week and complete at least 42 self-initiated texts per week, will receive a total of $\$ 285$.

\section{Measurement instruments}

Baseline and follow-up questionnaires: The baseline survey is a computer-assisted self-interview assessing sociodemographics, tobacco and ANDS use history, tobacco and ANDS use beliefs and cognitions, tobacco product and ANDS harm perceptions, alcohol use, and physical and mental health. An RA will be present in the interview room to answer participants' questions, but will not observe participants' responses without permission. The online follow-up survey will focus on current tobacco use, current ANDS use, nicotine dependence, interest in quitting smoking, and ANDS use beliefs/cognitions. The follow-up survey will be 
emailed to the participant or administered over the phone by an RA.

\section{EMA data}

EMA data collection will focus on cigarette/ANDS use and associated mood, craving and satisfaction. Responses will be collected via text message using participants' personal cell phones, eliminating the need to carry a study-specific device (figure 2). Each EMA data collection instance will take less than $1 \mathrm{~min}$ to complete. All EMA entries will be stamped with the time, date and geolocation (latitude/longitude) of the data report. The EMA data collection system will return an error message to prevent skipping items or entering out-of-range values. All data will be automatically pushed in real time and uploaded to Truth Initiative's secure, firewall protected geodatabase server via an encrypted representational state transfer (REST) application programmme interface (API).

\begin{tabular}{|c|c|c|}
\hline $\begin{array}{l}\bullet \bullet \circ 00 \text { Verizon LTE } \\
<\text { Messages }\end{array}$ & 9:21 AM & $\begin{array}{r}\text { C4 } 19 \% \square \text { Details } \\
\text { De }\end{array}$ \\
\hline
\end{tabular}

Sun, Nov 22, 12:39 PM

How is your mood right now? (1=Extremely bad 10=Extremely 12:39 PM good)

$1012: 40 \mathrm{PM}$

\begin{abstract}
Right now, how much do you want to smoke a cigarette? $(0=$ Not at all $9=$ Very, very much)
\end{abstract}

12:40 PM

Right now, how much do you
want to use an e-cig? ( $0=$ Not at
all $9=$ Very, very much)

0 12:40 PM

\begin{abstract}
Have you used a cig or e-cig in the past hour and forgotten to report it? (1=no,2=yes)
\end{abstract}

0 12:40 PM

Dav 3 Survev 2 complete

O Text Message

Figure 2 Example of the text message based EMA interface. EMA, ecological momentary assessment.
We will use two approaches to EMA data collection in the Moment Study: participant-initiated cigarette/ANDS use reports, and EMA system-generated random prompts. Participant-initiated EMA texts will collect information on cigarette consumption (weeks 1-3), ANDS consumption (weeks 2-3), satisfaction derived from the reported product use (weeks 1-3), and desire to use the opposite product (eg, desire to smoke after using an ANDS) (weeks 2-3). EMA system-generated random prompts will collect information on participants' immediate mood and craving for a cigarette, as well as assess missing cigarette/ANDS use reports and remind participants to report those behaviours. Items included in the participant-initiated and systemgenerated random texts are presented in box 1 .

For the random prompts, the EMA system will generate text messages sent to participants' phones six times each day, each falling within a prespecified 2.5-3 h time window. On weekdays, random texts will be delivered between 6:00 and 23:59; on weekends, random texts will be delivered between 9:00 and 23:59. The system will record the time it took the participant to respond to a prompt, time to completion and missed prompts. Participants will be encouraged to answer at least four random prompts a day and are incentivised to respond

Box 1 Items included in the participant-initiated and random ecological momentary assessment (EMA) prompts

Participant-initiated cigarette report

1. How many cigarettes did you just smoke?

2. Did you smoke inside ${ }^{\star}$ ? (1=no, 2=yes)

3. How many minutes ago did you finish smoking? Text 0 if you're still using it.

4. How satisfying was the cigarette? ( $0=$ not at all $9=$ extremely satisfying).

5. (Weeks 2-3 only) Right now, how much do you want to smoke an e-cig? ( $0=$ not at all, $9=$ =very, very much)

Participant-initiated ANDS report

1. About how many drags did you take on the e-cig?

2. Did you use the e-cig inside ${ }^{\star}$ ? ( $1=$ no, $\left.2=y e s\right)$

3. How many minutes ago did you finish using the e-cig? Text 0 if you're still using it.

4. How satisfying was the e-cig? $(0=$ not at all $9=$ extremely satisfying).

5. (Weeks 2-3 only) Right now, how much do you want to smoke a cigarette? ( $0=$ not at all, $9=$ very, very much)

EMA random prompts

1. How is your mood right now? (1=extremely bad $10=$ extremely good)

2. Right now, how much do you want to smoke a cigarette? ( $0=$ not at all $9=$ very, very much)

3. Have you used a cig or e-cig in the past hour and forgotten to report it? (1=no, 2=yes)

*'Indoors' is defined as a place surrounded by four walls and a ceiling. If windows are present, they must have glass and be able to be closed. An outdoor location is defined as a place missing at least one of four walls and/or is without a ceiling (eg, porch, rooftop, screened-in porch). 
to random prompts and initiate cigarette/ANDS use reports (see Compensation section). Random surveys will expire after 15 min of inactivity. Participants can selfinitiate tobacco use reports at any time of day. Text messages reminding participants to self-report tobacco use and complete random surveys will be delivered every day at 20:00.

\section{In-depth interviews}

An RA will conduct an in-depth interview with all participants at the weeks 1,2 and 3 office visits. The week 1 interview will focus on participants' social roles, the meaning and utility of smoking in their lives, and their perceptions of ANDS. In the weeks 2 and 3 in-depth interviews, the RA will investigate participants' understanding of the role of context, mood and craving on their product use. The RA will ask participants about their sensory and social experience using ANDS, and will ask participants to compare their ANDS experience to smoking their usual brand of cigarettes. For the week 3 interview, the RA will ask participants to discuss any different experiences with ANDS compared to the week before, others' reactions to ANDS use, perceived norms surrounding ANDS use, how these norms influenced their own use, and plans for ANDS use after the intensive monitoring period of the study ends. In-depth interviews will not exceed $30 \mathrm{~min}$.

\section{Geotracking}

We will track participants' physical location by installing an application ('Traccar') on their phones with their permission. Traccar turns a participant's cellphone into a personal GPS tracker and reports a participant's location (eg, latitude/longitude) at 5 min intervals. Similar to EMA data, geotracking data will be pushed in real time and uploaded to Truth Initiative's secure geodatabase server via an encrypted REST API. Using each participant's personal mobility map from the prior week's geotracking data, an RA will conduct a brief structured 'place' interview during the weeks 1-3 office visits using Esri's Collector for ArcGIS mapping application (Collector for ArcGIS [program]. 10.3.1 version. Redlands, California, 2016). This web-based mapping application provides the ability to synchronise the collection and geo-tagging of participants' 'place' locations relative to their previous geotracking data. An RA will display the participant's map and will geo-tag up to five locations with three or more points. Over the course of the three office visits, the participant may tag up to 15 locations. As a result of feedback from pilot testing, questions were added to the place survey to disentangle rules and norms concerning inside and outside cigarette smoking/ANDS use at the same location. Box 2 presents items from the place survey.

\section{Biomarkers}

Participants will provide two exhaled CO readings at the baseline and week 3 visits. CO readings will be collected
Box 2 Place survey items for indoor and outdoor locations

1. What is the place? Name it.

2. Is there a rule about smoking cigarettes here?

3. Has anyone smoked cigarettes here at all in the last month, as far as you know?

4. Have you smoked a cigarette here in the past week?

5 . Is there a rule about using e-cigarettes here?

6. Has anyone used an e-cigarette here at all in the last month, as far as you know?

7. Have you used an e-cigarette here in the past week?

using a calibrated Vitalograph BreathCO Carbon Monoxide Monitor and recorded by an RA. Participants will also provide two, $2 \mathrm{~mL}$ salivary cotinine samples at the baseline and week 3 visits using Salimetrics's passive drool method. ${ }^{26}$ The saliva sample will be immediately frozen at $0^{\circ}$ Fahrenheit and stored at Truth Initiative until ready for analysis, when it will be shipped frozen on dry ice to a lab at Virginia Commonwealth University and analysed via liquid chromatography mass spectrometry.

\section{Analyses}

Quantitative analyses

As the primary independent variables of interest, we will investigate how ANDS use affects cigarette consumption within-person. We will also examine how smoking-related and ANDS-related rules and norms surrounding indoor and outdoor product use influence cigarette and ANDS product use within-person. As secondary analyses, we will assess change in motivation to quit cigarettes (from the baseline vs follow-up surveys) and change in self-efficacy to quit cigarettes (also from the baseline vs follow-up) as a function of EMA-reported ANDS use. If statistical power allows, we will examine these questions betweenperson by race, menthol smoking status and gender. We will also examine the association between prior number of quit attempts at baseline and continued ANDS use at follow-up.

Our approach to analysing EMA data is based on published approaches using generalised linear mixed models. ${ }^{27-30}$ This class of models is useful for analysing EMA data because they allow multiple observations per subject, multiple levels of nesting (ie, observations within weeks within participants), multiple subject random effects, modelling of between-participant and within-participant variance as a function of covariates, and extension to non-normal outcomes (ie, dichotomous, ordinal, counts). Our primary approach will be a three-level mixed model treating observations (level 1) within observation week (level 2) within participants (level 3). The sample size was determined based on a preliminary power analysis and literature review of similar studies of cigarette smoking that suggested a sample size of at least 100 provides adequate power to provide scientific and statistical significance to the 
within-person results and will provide solid preliminary data for between-person results for subsequent research.

\section{Qualitative analyses}

We will examine how individuals understand the role of smoking restrictions on their ANDS use, and how cultural norms, self-concept, and gender roles surrounding cigarettes and ANDS affect product use. Our sample of 300 in-depth interviews (100 individuals interviewed 3 times) will yield rich data for within-person and between-person examinations. All in-depth interviews will be digitally recorded and transcribed verbatim by a professional transcription service. Transcripts will be cross-checked and de-identified by an RA. The principal investigator, project director, and RAs with experience in qualitative data thematic analysis will read and code the transcripts using NVivo 10 (QSR International Pty Ltd. NVivo qualitative data analysis software [program]. 10. Version, 2012) to facilitate organisation of coded data. A subset of the interviews will be independently read and reread by the principal investigator, project director and RAs to identify first-order codes based on the weeks 1-3 in-depth interview guides, such as 'motivation to quit cigarettes' and 'expectations about ANDS.' The entire data set will then be coded with these firstorder codes using Nvivo V.10. Study team members will read and code all transcripts independently. During the first-order coding process, coders will also independently create emerging codes which will be merged and then be grouped into higher-order themes in a careful and recursive decision-making process involving checking for emerging patterns and variability and consistency within and between coders. ${ }^{31}$ The thematically coded data will then be collated and reorganised through reading and rereading, allowing for a further refinement and review of themes.

\section{ETHICAL CONSIDERATION AND DISSEMINATION}

This study will be conducted according to USA and international standards of Good Clinical Practice (FDA guidance E6). This protocol and any amendments will be submitted to Chesapeake Institutional Review Board for formal approval to conduct the study. The decision of the IRB concerning the conduct of the study will be made in writing to the investigator. All personnel will be trained on study procedures, human protection issues and regulatory requirements. This study was funded by the National Institute on Drug Abuse of the US National Institutes of Health (1R21DA036472), registered at ClinicalTrials.gov as protocol number NCT02261363, and approved by the Chesapeake Institutional Review Board (Pro00008526).

A number of manuscripts related to the methods and results will be published from the collected data. These manuscripts will be published in a timely fashion as soon as data can be shared. To disseminate the findings of this project to the scientific community, data will be shared by presenting the findings at scientific meetings, US FDA CTP hearings, and Tobacco Product Scientific Advisory Committee meetings as appropriate, and by making relevant slide presentations available on request. Press releases will also be planned to disseminate significant findings to the lay community via Truth Initiative's Communications department.

\section{Author affiliations}

${ }^{1}$ Schroeder Institute for Tobacco Research and Policy Studies at Truth Initiative, Washington DC, USA

${ }^{2}$ Department of Health, Behavior, and Society, Johns Hopkins Bloomberg School of Public Health, Baltimore, Maryland, USA

${ }^{3}$ Department of Psychology, American University, Washington DC, USA

${ }^{4}$ George Washington University, Professional Psychology Program,

Washington DC, USA

${ }^{5}$ Department of Sociology and Anthropology, George Mason University, Fairfax, Virginia, USA

${ }^{6}$ New York University College of Global Public Health, New York, New York, USA

${ }^{7}$ Georgetown University Medical Center, Lombardi Comprehensive Cancer Center, Washington DC, USA

Acknowledgements At the time of the study, Sabrina L. Smiley was a Scholar with the HIV/AIDS, Substance Abuse, and Trauma Training Program (HA-STTP), at the University of California, Los Angeles; supported through an award from the National Institute on Drug Abuse (R25 DA035692).

Contributors All authors contributed to the conceptualisation of the Moment Study. JLP, SLS, EH, LFR, HE, and AA-R contributed to writing the paper. JLP is the principle investigator and SLS is the project director for the Moment Study.

Funding Research reported in this publication was supported by National Institute of Drug Abuse and FDA Center for Tobacco Products (CTP) under grant number R21DA036472. The content is solely the responsibility of the authors and does not necessarily represent the official views of the NIH or the Food and Drug Administration.

Competing interests None declared.

Ethics approval The study has been approved by Chesapeake IRB Pro00008526.

Provenance and peer review Not commissioned; peer reviewed for ethical and funding approval prior to submission.

Open Access This is an Open Access article distributed in accordance with the Creative Commons Attribution Non Commercial (CC BY-NC 4.0) license, which permits others to distribute, remix, adapt, build upon this work noncommercially, and license their derivative works on different terms, provided the original work is properly cited and the use is non-commercial. See: http:// creativecommons.org/licenses/by-nc/4.0/

\section{REFERENCES}

1. King BA, Alam S, Promoff G, et al. Awareness and ever-use of electronic cigarettes among U.S. adults, 2010-2011. Nicotine Tob Res 2013;15:1623-7.

2. King BA, Patel R, Nguyen $\mathrm{KH}$, et al. Trends in awareness and use of electronic cigarettes among U.S. adults, 2010-2013. Nicotine Tob Res 2015;17:219-27.

3. Delnevo CD, Giovenco DP, Steinberg MB, et al. Patterns of electronic cigarette use among adults in the United States. Nicotine Tob Res 2016;18:715-19.

4. Pearson JL, Richardson A, Niaura RS, et al. e-Cigarette awareness, use, and harm perceptions in US adults. Am J Public Health 2012;102:1758-66.

5. Adkison SE, O'Connor RJ, Bansal-Travers M, et al. Electronic nicotine delivery systems: international tobacco control four-country survey. Am J Prev Med 2013;44:207-15. 
6. Dawkins L, Turner J, Roberts A, et al. 'Vaping' profiles and preferences: an online survey of electronic cigarette users. Addiction 2013;108:1115-25.

7. Etter JF. Electronic cigarettes: a survey of users. BMC Public Health 2010;10:231.

8. Farsalinos KE, Romagna G, Tsiapras D, et al. Characteristics, perceived side effects and benefits of electronic cigarette use: a worldwide survey of more than 19,000 consumers. Int J Environ Res Public Health 2014;11:4356-73.

9. Goniewicz ML, Lingas EO, Hajek P. Patterns of electronic cigarette use and user beliefs about their safety and benefits: an internet survey. Drug Alcohol Rev 2013;32:133-40.

10. Kralikova E, Novak J, West O, et al. Do e-cigarettes have the potential to compete with conventional cigarettes?: A survey of conventional cigarette smokers' experiences with e-cigarettes. Chest 2013;144:1609-14.

11. McNeill A, Brose L, Calder R, et al. E-cigarettes: an evidence update -a report commissioned by Public Health England. London, UK: Public Health England, 2015

12. McRobbie $\mathrm{H}$, Bullen $\mathrm{C}$, Hartmann-Boyce $\mathrm{J}$, et al. Electronic cigarettes for smoking cessation and reduction. Cochrane Database Syst Rev 2014;12:CD010216.

13. Caponnetto $P$, Campagna D, Cibella F, et al. EffiCiency and Safety of an eLectronic cigAreTte (ECLAT) as Tobacco Cigarettes Substitute: a prospective 12-month randomized control design study PLOS ONE 2013;8:e66317.

14. Biener L, Hargraves JL. A longitudinal study of electronic cigarette use in a population-based sample of adult smokers: association with smoking cessation and motivation to quit. Nicotine Tob Res 2015;17:127-33.

15. Brose LS, Hitchman SC, Brown J, et al. Is the use of electronic cigarettes while smoking associated with smoking cessation attempts, cessation and reduced cigarette consumption? A survey with a 1-year follow-up. Addiction 2015;110:1160-8.

16. Bullen $\mathrm{C}$, Howe $\mathrm{C}$, Laugesen $\mathrm{M}$, et al. Electronic cigarettes for smoking cessation: a randomised controlled trial. The Lancet 2013;382:1629-37.

17. Brown J, Beard E, Kotz D, et al. Real-world effectiveness of e-cigarettes when used to aid smoking cessation: a cross-sectional population study. Addiction 2014;109:1531-40.

18. Hitchman SC, Brose LS, Brown J, et al. Associations between e-cigarette type, frequency of use, and quitting smoking: findings from a longitudinal online panel survey in Great Britain. Nicotine Tob Res 2015;17:1187-94.

19. Dockrell M, Morrison R, Bauld L, et al. E-cigarettes: prevalence and attitudes in Great Britain. Nicotine Tob Res 2013;15:1737-44.

20. H.R. 1256: Family Smoking Prevention and Tobacco Control Act. 21 USC. 1st Session of the 111th Congress ed. USA: In GovTrack.us (database of federal legislation). 2009.

21. Villanti AC, Vargyas EJ, Niaura RS, et al. Food and Drug Administration regulation of tobacco: integrating science, law, policy, and advocacy. Am J Public Health 2011:101:1160-2.

22. Bullen $\mathrm{C}$, McRobbie $\mathrm{H}$, Thornley $\mathrm{S}$, et al. Effect of an electronic nicotine delivery device (e-cigarette) on desire to smoke and withdrawal, user preferences and nicotine delivery: randomised cross-over trial. Tob Control 2010;19:98-103.

23. Etter JF, Bullen C. Electronic cigarette: users profile, utilization, satisfaction and perceived efficacy. Addiction 2011;106: 2017-28.

24. Goniewicz ML, Lingas EO, Hajek P. Patterns of electronic cigarette use and user beliefs about their safety and benefits: an Internet survey. Drug Alcohol Rev 2013;32:133-40.

25. Siegel MB, Tanwar KL, Wood KS. Electronic cigarettes as a smoking-cessation: tool results from an online survey. Am J Prev Med 2011;40:472-5.

26. Salimetrics L.L.C., Salimetrics Europe. syncii://Capella 2012.pdf

27. Hedeker D, Mermelstein RJ, Berbaum ML, et al. Modeling mood variation associated with smoking: an application of a heterogeneous mixed-effects model for analysis of ecological momentary assessment (EMA) data. Addiction 2009;104: 297-307.

28. Hedeker D, Mermelstein RJ, Demirtas H. An application of a mixed-effects location scale model for analysis of Ecological Momentary Assessment (EMA) data. Biometrics 2008;64: 627-34.

29. Hedeker D, Mermelstein RJ, Demirtas H. Modeling between-subject and within-subject variances in ecological momentary assessment data using mixed-effects location scale models. Stat Med 2012;31:3328-36.

30. Kirchner TR, Shiffman S, Wileyto EP. Relapse dynamics during smoking cessation: recurrent abstinence violation effects and lapse-relapse progression. J Abnorm Psychol 2012;121:187-97.

31. Braun V, Clarke V. Using thematic analysis in psychology. Qual Res Psychol 2006;3:77-101. 